Uso de las TIC en las PYMIE del sector industrial del Área Metropolitana de Bucaramanga

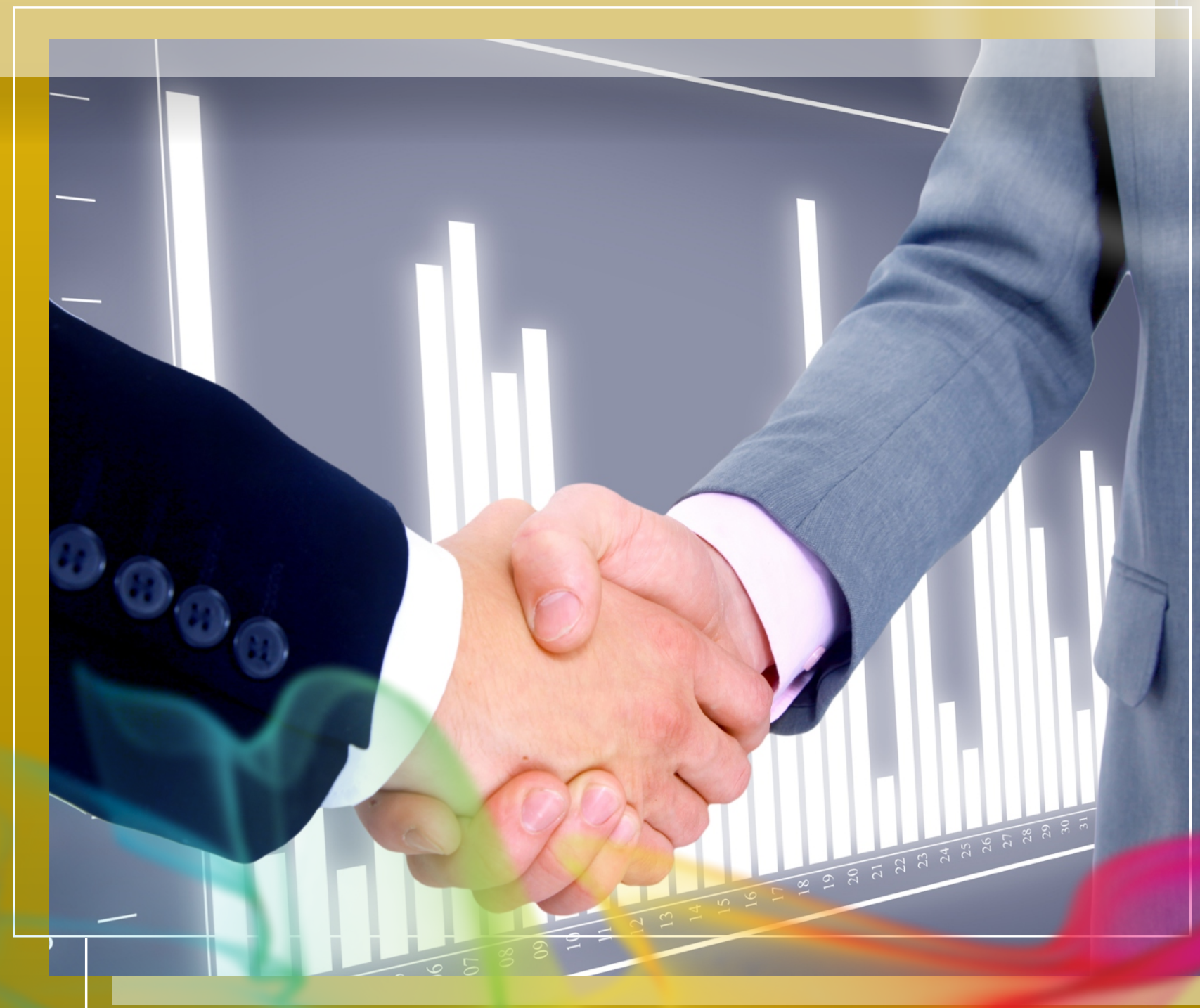




\title{
Uso de las TIC en las PYME del sector industrial del Área Metropolitana de Bucaramanga ${ }^{1}$
}

\section{Use of ICT in small and medium scale enterprises of the industrial sector of the metropolitan area of Bucaramanga}

\author{
Germán Manuel Argüello López², \\ Corporación Universitaria de Investigación y Desarrollo (UDI) Bucaramanga, Colombia.
}

Artículo recibido en julio de 2015, artículo aceptado en agosto de 2015.

Citación del artículo: Argüello, G. (2015). Uso de las TIC en las PYME del sector industrial del Área Metropolitana de Bucaramanga. I+D Revista de Investigaciones, 6(2), 125-136.

\section{Resumen}

Numerosas investigaciones han demostrado que las TIC pueden aumentar la productividad de las empresas; en este sentido, el presente estudio -cuyo enfoque es cuantitativo no experimental de corte transversal y de alcance correlaccional/causal-- fue desarrollado con el fin de satisfacer la necesidad de poseer información actualizada sobre el uso de las TIC en las pyme del sector industrial del Área Metropolitana de Bucaramanga (AMB). La muestra se compuso de 35 empresas medianas y 182 empresas pequeñas que fueron seleccionadas mediante muestreo aleatorio estratificado. El instrumento de recolección de datos fue un cuestionario que se aplicó mediante entrevista personal. Algunas conclusiones relevantes de este estudio señalan, para la muestra seleccionada, que el uso de software especializado es muy limitado en las pyme del sector industrial, que el uso de las distintas herramientas ofimáticas depende del subsector analizado, que la generación de páginas web propias, el uso de redes sociales y las plataformas electrónicas para interactuar con los clientes y proveedores es aún deficitario en la industria local y no está acorde con la necesidad que tienen de ser más productivas y competitivas

Palabras clave: Industria, TIC, PYME.

${ }^{1}$ Artículo de investigación, de enfoque cualitativo, resultado de un proyecto de investigación desarrollado en el grupo de investigación Sinergia, financiado por la Corporación Universitaria de Investigación y Desarrollo (UDI) Bucaramanga, Colombia. Dirección: Calle 9 No. 23-55, PBX: 6352525. Fecha de inicio: febrero de 2014, fecha de terminación: junio de 2015.

${ }^{2}$ Ingeniero Industrial, UIS. Especialista en Entornos Virtuales de Aprendizaje, Universidad de Panamá. Docente investigador del grupo: Sinergia. Corporación Universitaria de Investigación y Desarrollo (UDI) Bucaramanga, Colombia. Dirección: Calle 9 No. 23-55, PBX: 6352525. Correo electrónico institucional: garguello1@udi.edu.co. 


\section{Abstract}

Numerous studies have shown that ICT can increase the productivity of enterprises. This study was developed in order to satisfy the need to possess information updated about the use of ICT in SMEs in the industrial sector of the Metropolitan Area of Bucaramanga (AMB). This study was a non-experimental cross-sectional quantitative approach and correlaccional / causal scope. The sample was composed of 35 mediumsized companies and 182 small businesses that were selected by stratified random sampling. The instrument of compilation of information was a questionnaire that was applied by personal interview. Some important conclusions of this study are: i) The use of ERP software $(95 \% \mathrm{CI}$ : $9.98 \% ; 19.38 \%)$, design software (95\% CI: $7.24 \% ; 15.70 \%)$ or another kind of specialized software (95\% CI: $7.86 \% ; 16.55 \%$ ) is limited. ii) Most SMEs do not have a formal policy to use social media (95\% CI: 79,42\%; 84,64\%). iii) The use of an electronic platform for selling is uncommon (95\% CI: $12,84 \% ; 23,10 \%$ ) and the percentage of Internet sales is not significant (95\% CI: 20,25\%; 25,01\%). iv). The majority of SMEs $(61.93 \%)$ spent less than 5 million in ICT in 2013.

Keywords: Industry, ICT, SMEs.

\section{Introducción}

El uso de las TIC contribuye al logro del posicionamiento estratégico de una empresa siempre y cuando contribuya a la creación de una propuesta de valor única y facilite la interrelación entre las actividades(Porter, 2011).
La importancia de las TIC para las pequeñas y medianas empresas (pyme) de un sector varía según el contexto regional y su relación con el entorno mundial (Rodríguez Melo, 2011). Estos son algunos estudios sobre el impacto de uso de las TIC en las pyme:

Una investigación del Centro Internacional de Investigaciones para el Desarrollo (CIID) en colaboración con el BID concluyó que las empresas que invierten en TIC pueden ahorrar hasta un $10 \%$ en costos y a su vez pueden incrementar las ventas en el mismo porcentaje '(CaZorzi, 2011).

Una de las conclusiones de un estudio adelantado por el Computing Technology Industry Association - CompTIA en Asociacion con Nathan Associates y Sallstrom Consulting fue que las economías con niveles altos de uso de TIC experimentan una productividad laboral promedio siete veces más elevada que la de los países con bajo uso de las TIC(CompTIA, 2007).

Una revisión de estudios empíricos desarrollada por Cardona, Kretschmer y Strobel (2013) sobre el impacto del uso de las TIC en la productividad concluyó que el impacto no solo es significativo y positivo, sino que también aumenta con el tiempo, aclarando que este efecto no es solo el resultado de la implementación de las TIC, debiéndose considerar también las competencias y estructuras empresariales.

Un estudio desarrollado por Colombo, Croce y Grilli (2013) sobre el efecto del uso del Internet de banda ancha en la productividad en las pyme italianas concluyó que la obtención de un impacto positivo en el uso de la banda ancha 
depende de la implementación de aplicaciones dirigidas a mejorar la gestión de actividades específicas de la empresa y que dicha implementación vaya acompañada de cambios estratégicos y organizativos complementarios.

Un estudio econométrico desarrollado por Martin, Ciovica y Cristescu (2013) en pyme de Rumania concluyó que a medida que las empresas crecen se hace más complejo administrar y controlar los flujo de información y comunicación, siendo la adopción y el desarrollo de las TIC el medio idóneo para superar esta dificultad.

La implementación de sistemas de información es un elemento de apalancamiento competitivo y de aumento del rendimiento operativo y financiero; según Does Reis y Do Carmo (2014) entre los factores que favorecen la implementación de un sistema de información destacan la usabilidad y la percepción de utilidad y entre los factores que la obstaculizan están el deficiente entrenamiento y soporte técnico ofrecido por el proveedor así como el bajo nivel de transferencia tecnológica del proveedor al comprador.

Un estudio empírico desarrollado por Gálvez (2014) en las mipyme de Colombia concluyó que aunque el grado de uso de las TIC es apenas aceptable, la implementación de las herramientas y prácticas asociadas a las TIC influyen positivamente en el nivel de innovación de las mipyme, teniendo mayor efecto positivo en la innovación en productos o servicios y en la gestión.
Un estudio empírico desarrollado por Gálvez, Riascos y Contreras (2014) en las mipyme de Colombia concluyó que aunque la disponibilidad y utilización las TIC en ambiente web (comercio electrónico, mercadeo electrónico, redes sociales, etc.) es apenas aceptable, su implementación influye positivamente en el rendimiento global de la empresa y con mayor grado en su participación en el mercado, la rentabilidad y la productividad, independientemente del tamaño y la edad de la empresa.

Un estudio sobre uso de las TIC en sectores estratégicos para el país en un contexto de globalización, desarrollado por el Centro de Estrategia y Competitividad de la Universidad de los Andes, concluyó que los principales inconvenientes percibidos por las pyme para la implementación de las TIC eran la falta de recursos y las limitaciones de acceso al crédito (Rodríguez Melo, 2011).

El sector industrial de Santander representó el 28,5 \% del PIB departamental del 2013 a precios corrientes, constituyéndose como el segundo sector en importancia después de los servicios. (Cámara de Comercio de Bucaramanga, 2015). No existen estudios específicos sobre el uso de las TIC por parte de las pyme del sector industrial del Área Metropolitana de Bucaramanga.

\section{Método}

\section{Tipo de estudio}

La investigación tuvo un enfoque cuantitativo no experimental de corte transversal de alcance correlaccional/causal. 


\section{Participantes y datos}

La población meta seleccionada fueron las pyme del sector industrial del AMB registradas en la Cámara de Comercio de Bucaramanga, 498 pyme: 83 medianas y 415 pequeñas. La técnica de muestreo utilizada fue el muestreo aleatorio estratificado y con reemplazo, tomando como estratos el tamaño de las empresas (pequeña empresa y mediana empresa). Considerando que no existían estudios previos y estableciendo un nivel de confianza del $95 \%$ y un margen de error del $5 \%$, el tamaño de la muestra resultó ser 35 empresas medianas y 182 empresas pequeñas.

Las variables estudiadas de las pyme fueron las siguientes: 1. Sector. 2. Número de empleados. 3. Años de existencia. 4. Número de computadores. 5. Porcentaje de empleados que utilizan computador al menos una vez por semana. 6. Herramientas ofimáticas utilizadas. 7. Utilización de programas informáticos especializados. 8. Funciones empresariales apoyadas por programas informáticos especializados. 9. Disponibilidad de conexión a Internet. 10. Porcentaje de empleados que utilizan Internet al menos una vez por semana. 11. Velocidad de navegación.12. Usos de Internet. 13. Disponibilidad de página web. 14. Servicios disponibles en la página web. 15. Utilización de medios sociales. 16. Tipos de medios sociales utilizados. 17. Fines de la utilización de los medios sociales. 18. Disponibilidad de política formal para uso de medios sociales. 19. Percepción del nivel de importancia de uso de las TIC para desarrollo del negocio. 20. Utilización de plataforma electrónica para vender o comprar. 21. Porcentaje de ventas por internet en el último año. 22. Porcentaje de compras por internet en el último año. 23. Inversión en TIC en el último año.

\section{Materiales e instrumentos}

El instrumento de recolección de datos utilizado fue un cuestionario diseñado con base en la operacionalización de las variables y el respectivo desarrollo de los ítems. Una versión preliminar del cuestionario se aplicó de manera piloto a veinte PYME, lo cual condujo a la realización de ajustes. El cuestionario final tenía veintitrés 3 preguntas de distintos tipos: sí y no, respuesta numérica, opción múltiple con única respuesta, opción múltiple con múltiple respuesta y dos preguntas abiertas.

\section{Procedimiento}

Mediante generación de números aleatorios se seleccionaron del listado de la Cámara de Comercio las pyme dónde se iba a aplicar el cuestionario. Este se aplicó de manera personal con el apoyo de estudiantes de Ingeniería Industrial vinculados como auxiliares de investigación, quienes fueron debidamente capacitados. El trabajo de campo se realizó desde el 19 de agosto hasta el 24 de septiembre de 2014 en horario de oficina. El cuestionario fue contestado por el jefe de sistemas, el jefe de tecnologías, el gerente-propietario u otro cargo con acceso a la información requerida. Los encuestados firmaron un consentimiento informado autorizando la utilización de la información recolectada para fines de la investigación.

Los cuestionarios se revisaron con el fin de verificar su correcto diligenciamiento y detectar inconsistencias. Los datos se tabularon con la ayuda de la herramienta Google docs para formularios y la información de los cuestionarios se confrontó con la información registrada. 
La composición de la muestra por subsectores resulto ser la siguiente: calzado y marroquinería $38,25 \%$, muebles y productos de madera $12,44 \%$, confecciones $11,521 \%$, máquinas, herramientas $\mathrm{u}$ otros productos metálicos $10,60 \%$, alimentos procesados $9,68 \%$, autopartista 5,07\%, plásticos y caucho 3,69\%, artes gráficas 3,23\%, materiales de construcción y cerámica $3,23 \%$ y otros $2,30 \%$. Para la estimación de los parámetros poblacionales fue necesario realizar los cálculos que permitieran armonizar los pesos de la muestra con los de la población, mediante la respectiva ponderación.

\section{Resultados}

En la Tabla 1 se aprecian la media, el error estándar y el intervalo de confianza del $95 \%$ para las principales variables del estudio.

Tabla 1. Media, error estándar e intervalo de confianza del $95 \%$ para las principales variables

\begin{tabular}{|c|c|c|c|}
\hline Variable & Media & $\begin{array}{l}\text { Error } \\
\text { estándar }\end{array}$ & IC $95 \%$ \\
\hline $\begin{array}{l}\text { Número promedio de computadores por } \\
\text { empresa }\end{array}$ & 10,46 & 1,06 & $8,37-12,55$ \\
\hline $\begin{array}{l}\text { Porcentaje promedio de empleados que } \\
\text { utiliza el computador al menos una vez a la } \\
\text { semana }\end{array}$ & $25,40 \%$ & $1,73 \%$ & $22,02 \%-28,78 \%$ \\
\hline $\begin{array}{l}\text { Porcentaje de pyme que utilizan software } \\
\text { especializado }\end{array}$ & $58,99 \%$ & $3,23 \%$ & $52,65 \%-65,32 \%$ \\
\hline Porcentaje de pyme con conexión a internet & $97,70 \%$ & $1,02 \%$ & $95,70 \%-99,69 \%$ \\
\hline $\begin{array}{l}\text { Porcentaje de empleados que utilizan } \\
\text { internet al menos una vez a la semana }\end{array}$ & $22,33 \%$ & $1,67 \%$ & $19,06 \%-25,60 \%$ \\
\hline Porcentaje de pyme que tienen página web & $51,15 \%$ & $3,25 \%$ & $44,79 \%-57,51 \%$ \\
\hline $\begin{array}{l}\text { Porcentaje de pyme que utilizan medios } \\
\text { sociales }\end{array}$ & $57,60 \%$ & $3,37 \%$ & $51,00 \%-64,21 \%$ \\
\hline $\begin{array}{l}\text { Porcentaje de pyme que tienen una política } \\
\text { formal para la utilización de medios sociales }\end{array}$ & $17,97 \%$ & $2,61 \%$ & $12,86 \%-23,09 \%$ \\
\hline $\begin{array}{c}\text { Porcentaje de pyme que utilizan una } \\
\text { plataforma electrónica para comprar o } \\
\text { vender. }\end{array}$ & $17,97 \%$ & $2,62 \%$ & $12,84 \%-23,10 \%$ \\
\hline $\begin{array}{l}\text { Porcentaje de ventas por internet con } \\
\text { respecto al total de ventas de las pyme que } \\
\text { venden por internet. }\end{array}$ & $22,63 \%$ & $1,21 \%$ & $20,25 \%-25,01 \%$ \\
\hline $\begin{array}{l}\text { Porcentaje de compras por internet con } \\
\text { respecto al total de compras de las pyme que } \\
\text { compran por internet. }\end{array}$ & $17,72 \%$ & $1,38 \%$ & $15,01 \%-20,44 \%$ \\
\hline
\end{tabular}


La Tabla 2 presenta las pruebas de hipótesis de igualdad de medias de algunas variables para distintos subsectores. Se destaca que en cuanto a porcentaje de ventas y compras por Internet no hay ningún subsector con un mejor desempeño a los demás.

Tabla 2. Prueba de hipótesis de igualdad de medias de algunas variables para distintos subsectores

\begin{tabular}{|c|c|c|c|}
\hline Hipótesis & $\begin{array}{c}\text { Criterio de rechazo } \\
\text { de } H o(\alpha=0,05)\end{array}$ & Valor P & LSD de Fisher \\
\hline $\begin{array}{l}\text { El número promedio de } \\
\text { computadores es igual para los } \\
\text { distintos subsectores. }\end{array}$ & $\begin{array}{c}\mathrm{F}=10,70>0.05,8,178=1,99 \\
\text { Se rechaza }\end{array}$ & $3,05 \times 10^{-12}$ & $\begin{array}{l}\text { Menor que el promedio: } \\
\text { calzado, muebles, } \\
\text { confecciones, autopartista. } \\
\text { Mayor que el promedio: } \\
\text { plástico y caucho }\end{array}$ \\
\hline $\begin{array}{l}\text { El porcentaje de utilización del } \\
\text { computador es igual para los } \\
\text { distintos subsectores. }\end{array}$ & $\begin{array}{c}\mathrm{F}=12,23>0.05,8,177=1,99 \\
\text { Se rechaza }\end{array}$ & $7,17 \times 10^{-14}$ & $\begin{array}{l}\text { Menor que el promedio: } \\
\text { calzado, muebles, artes } \\
\text { gráficas. } \\
\text { Mayor que el promedio: } \\
\text { Autopartista }\end{array}$ \\
\hline $\begin{array}{l}\text { El porcentaje de utilización de } \\
\text { Internet es igual para los } \\
\text { distintos subsectores. }\end{array}$ & $\begin{array}{c}\mathrm{F}=5,997>0.05,8,178=1,99 \\
\text { Se rechaza }\end{array}$ & $8,14 \times 10^{-7}$ & $\begin{array}{c}\text { Menor que el promedio: } \\
\text { calzado, plásticos y caucho. } \\
\text { Mayor que el promedio: } \\
\text { N.A. }\end{array}$ \\
\hline $\begin{array}{l}\text { El porcentaje de ventas por } \\
\text { Internet es igual para los } \\
\text { distintos subsectores. }\end{array}$ & $\begin{array}{c}\mathrm{F}=0,6653<0.05,5,26=2,587 \\
\text { No se rechaza }\end{array}$ & - & - \\
\hline $\begin{array}{l}\text { El porcentaje de compras por } \\
\text { Internet es igual para los } \\
\text { distintos subsectores. }\end{array}$ & $\begin{array}{c}\mathrm{F}=0,498<0.05,5,19=2,74 \\
\text { No se rechaza }\end{array}$ & - & - \\
\hline
\end{tabular}

Fuente: El autor.

La Tabla 3 presenta la prueba de independencia de algunas variables con respecto al tamaño de las PYME. El análisis de los residuos estandarizados de estas pruebas permitió concluir, con un nivel de significancia del $1 \%$, que las empresas medianas hacen mayor uso de software especializado (incluyendo software ERP), tienen mayores velocidades de conexión, disponen en mayor proporción de página web y hacen mayores niveles de inversión en TIC. No obstante, las empresas medianas no se destacan con respecto a las empresas pequeñas en cuanto a uso de medios sociales y utilización de alguna plataforma de comercio electrónico. 
Tabla 3. Prueba de independencia de algunas variables con respecto al tamaño de las pyme

\begin{tabular}{|c|c|c|}
\hline Hipótesis & $\begin{array}{l}\text { Criterio de rechazo } \\
\text { de } \mathrm{Ho}(\alpha=0,05)\end{array}$ & Valor $\mathbf{P}$ \\
\hline $\begin{array}{l}\text { La utilización de software especializado es } \\
\text { independiente del tamaño de la empresa. }\end{array}$ & $\begin{array}{c}\chi_{0}^{2}=15,098>\chi_{0.05,64}^{2}=3,841 \\
\text { Se rechaza }\end{array}$ & $1 \times 10^{-4}$ \\
\hline $\begin{array}{l}\text { La utilización de software ERP es } \\
\text { independiente del tamaño de la empresa. }\end{array}$ & $\begin{array}{c}\chi_{0}^{2}=31,833>\chi_{0.05,1}^{2}=3,841 \\
\text { Se rechaza }\end{array}$ & $1 \times 10^{-4}$ \\
\hline $\begin{array}{l}\text { La velocidad de conexión es independiente } \\
\text { del tamaño de la empresa. }\end{array}$ & $\begin{array}{c}\chi_{0}^{2}=61,92>\chi_{0.05,4}^{2}=9,488 \\
\text { Se rechaza }\end{array}$ & $1 \times 10^{-4}$ \\
\hline $\begin{array}{l}\text { La tenencia de página web es } \\
\text { independiente del tamaño de la empresa. }\end{array}$ & $\begin{array}{c}\chi_{0}^{2}=19,95>\chi_{0.05,1}^{2}=3,841 \\
\text { Se rechaza }\end{array}$ & $1 \times 10^{-4}$ \\
\hline $\begin{array}{l}\text { La utilización de medios sociales es } \\
\text { independiente del tamaño de la empresa. }\end{array}$ & $\begin{array}{c}\chi_{0}^{2}=0,098<\chi_{0.05,1}^{2}=3,841 \\
\text { No se rechaza }\end{array}$ & - \\
\hline $\begin{array}{l}\text { El nivel de importancia que se da al uso de } \\
\text { medio sociales es independiente del } \\
\text { tamaño de la empresa. }\end{array}$ & $\begin{array}{c}\chi_{0}^{2}=1,557<\chi_{0.05,4}^{2}=9,488 \\
\text { No se rechaza }\end{array}$ & - \\
\hline $\begin{array}{l}\text { La existencia de una política formal de uso } \\
\text { de los medios sociales es independiente del } \\
\text { tamaño de la empresa. }\end{array}$ & $\begin{array}{c}\chi_{0}^{2}=1,598<\chi_{0.05,1}^{2}=3,841 \\
\text { No se rechaza }\end{array}$ & - \\
\hline $\begin{array}{l}\text { La utilización de una plataforma de } \\
\text { comercio electrónico es independiente del } \\
\text { tamaño de la empresa. }\end{array}$ & $\begin{array}{c}\chi_{0}^{2}=0,09<\chi_{0.05,1}^{2}=3,841 \\
\text { No se rechaza }\end{array}$ & - \\
\hline $\begin{array}{l}\text { El nivel de inversión en TIC en el último } \\
\text { año es independiente del tamaño de la } \\
\text { empresa. }\end{array}$ & $\begin{array}{c}\chi_{0}^{2}=45,887>\chi_{0.05,4}^{2}=9,488 \\
\text { Se rechaza }\end{array}$ & $1 \times 10^{-4}$ \\
\hline
\end{tabular}

Fuente: El autor.

La tabla 4 presenta la prueba de independencia de algunas variables con respecto al subsector de las pyme. 
Tabla 4. Prueba de independencia de algunas variables con respecto al subsector

\begin{tabular}{|c|c|c|}
\hline Hipótesis & $\begin{array}{c}\text { Criterio de rechazo } \\
\text { de } \mathrm{Ho}(\alpha=\mathbf{0 , 0 5})\end{array}$ & Valor $\mathbf{P}$ \\
\hline $\begin{array}{l}\text { La utilización de las herramientas ofimáticas es } \\
\text { independiente del subsector. }\end{array}$ & $\begin{array}{c}\chi_{0}^{2}=374,85>\chi_{0.05,64}^{2}=50,998 \\
\text { Se rechaza }\end{array}$ & $1 \times 10^{-4}$ \\
\hline $\begin{array}{l}\text { La utilización de software especializado es } \\
\text { independiente del subsector. }\end{array}$ & $\begin{array}{c}\chi_{0}^{2}=15,555<\chi_{0.05,9}^{2}=16,919 \\
\text { No se rechaza }\end{array}$ & - \\
\hline $\begin{array}{l}\text { La utilización de software ERP es } \\
\text { independiente del subsector. }\end{array}$ & $\begin{array}{c}\chi_{0}^{2}=12,134<\chi_{0.05,9}^{2}=16,919 \\
\text { No se rechaza }\end{array}$ & - \\
\hline $\begin{array}{l}\text { La velocidad de conexión es independiente del } \\
\text { subsector. }\end{array}$ & $\begin{array}{c}\chi_{0}^{2}=71,64>\chi_{0.05,36}^{2}=50,998 \\
\text { Se rechaza }\end{array}$ & $3,8 \times 10^{-4}$ \\
\hline $\begin{array}{l}\text { Los usos de Internet son independientes del } \\
\text { subsector. }\end{array}$ & $\begin{array}{c}\chi_{0}^{2}=358,55>\chi_{0.05,88}^{2}=110,9 \\
\text { Se rechaza }\end{array}$ & $1 \times 10^{-4}$ \\
\hline $\begin{array}{l}\text { La tenencia de página web es independiente del } \\
\text { subsector. }\end{array}$ & $\begin{array}{c}\chi_{0}^{2}=19,384>\chi_{0.05,9}^{2}=16,919 \\
\text { Se rechaza }\end{array}$ & 0,0221 \\
\hline $\begin{array}{l}\text { Los servicios disponibles en la página web son } \\
\text { independientes del subsector. }\end{array}$ & $\begin{array}{c}\chi_{0}^{2}=717,19>\chi_{0.05,72}^{2}=102,816 \\
\text { Se rechaza }\end{array}$ & $1 \times 10^{-4}$ \\
\hline $\begin{array}{l}\text { La utilización de medios sociales es } \\
\text { independiente del subsector. }\end{array}$ & $\begin{array}{c}\chi_{0}^{2}=2,88<\chi_{0.05,9}^{2}=16,919 \\
\text { No se rechaza }\end{array}$ & - \\
\hline $\begin{array}{l}\text { El nivel de importancia que se da al uso de } \\
\text { medio sociales es independiente del subsector. }\end{array}$ & $\begin{array}{c}\chi_{0}^{2}=32,021<\chi_{0.05,36}^{2}=50,998 \\
\text { No ser rechaza }\end{array}$ & - \\
\hline $\begin{array}{l}\text { La existencia de una política formal de uso de } \\
\text { los medios sociales es independiente del } \\
\text { subsector. }\end{array}$ & $\begin{array}{c}\chi_{0}^{2}=8,159<\chi_{0.05,9}^{2}=16,919 \\
\text { No se rechaza }\end{array}$ & - \\
\hline $\begin{array}{l}\text { La utilización de una plataforma de comercio } \\
\text { electrónico es independiente del subsector. }\end{array}$ & $\begin{array}{c}\chi_{0}^{2}=8,91<\chi_{0.05,9}^{2}=16,919 \\
\text { No se rechaza }\end{array}$ & - \\
\hline $\begin{array}{l}\text { El nivel de inversión en TIC en el último año es } \\
\text { independiente del subsector. }\end{array}$ & $\begin{array}{c}\chi_{0}^{2}=67,38>\chi_{0.05,36}^{2}=50,998 \\
\text { Se rechaza }\end{array}$ & 0,0012 \\
\hline
\end{tabular}

El análisis de los residuos estandarizados de estas pruebas permitió concluir, con un nivel de significancia del $1 \%$, que ningún subsector se destaca con respecto a los otros en cuanto a la utilización de software especializado (incluyendo software ERP), medios sociales o plataforma de comercio electrónico. No obstante, en cuanto a las siguientes variables sí hay subsectores que se destacan:

Los subsectores Autopartes y Artes gráficas se caracterizan por el uso de Project y los subsectores Confecciones y Alimentos procesados son los que menos hacen uso de las herramientas ofimáticas.

Los subsectores Alimentos procesados y Materiales de construcción se caracterizan por tener una velocidad de conexión de 20 o más $\mathrm{Mb} / \mathrm{seg}$.

En cuanto a los usos de Internet, el subsector autopartista se caracteriza por el uso de aplicaciones, procesos de contratación y venta de productos online; el subsector Materiales de construcción por capacitación de empleados y el subsector Máquinas y productos metálicos por el uso de aplicaciones. 
En cuanto a disponibilidad de página web, las pyme del subsector Calzado se caracterizan por no contar con página web y las pyme de los subsectores Máquinas y productos metálicos y Materiales de construcción por tener página.

En cuanto a servicios disponibles en la página web el subsector Calzado se caracteriza por ofrecer el servicio de realización de pedidos; los subsectores Artes gráficas y Alimentos procesados por ofrecer seguimiento online de pedidos; los subsectores Máquinas y productos metálicos, Alimentos procesados y Plásticos y caucho por publicar ofertas laborales y recibir hojas de vida y los subsectores Artes gráficas y Confecciones por recibir pagos en línea.

En cuanto a inversión en tecnologías, las pyme del subsector Calzado se caracterizaron por invertir menos de cinco millones, las del subsector Máquinas y productos metálicos por invertir entre cinco y menos de veinte millones de pesos y las del subsector Alimentos procesados por invertir más de doscientos millones de pesos.

Otros resultados importantes del análisis de otras preguntas del estudio son:

La gran mayoría de la pyme utiliza Excel (IC $95 \%$ : 92,49\%; 98,12\%) y Word (IC 95\%: $91,30 \% ; 97,43 \%$ ) y aproximadamente la mitad utiliza Power Point (IC 95 \%: 43,58 \%; 56,89 \%) y Outlook (IC 95\%: 50,22\%; 63,40\%). La utilización de Microsoft Access (IC 95\%: $6,67 \%$; 14,93 \%) y Microsoft Project (IC 95\%: $1,55 \% ; 6,90 \%$ ) es muy limitada.

El software especializado de mayor utilización es de tipo contable (IC $95 \%$ : 28,54 \%; $41,19 \%$ ), pero algunas empresas reportaron uso de soluciones ERP (IC $95 \%$ : 9,98\%; 19,38\%), software de diseño (IC $95 \%: 7,24 \% ; 15,70 \%$ ) u otro tipo de software (IC $95 \%: 7,86 \% ; 16,55 \%$ ).

La gran mayoría de la pyme utiliza internet para recibir y enviar correos electrónicos (IC $95 \%$ : 96,95\%; $100 \%$ ) y para buscar información (IC 95\%: 87,01\%; 94,81\%), aproximadamente dos de tres lo utilizan para hacer transacciones bancarias (IC 95\%: 59,11\%; $71,99 \%$ ) y aproximadamente la mitad lo utiliza para brindar servicio al cliente (IC 95\%: $47,79 \% ; 61,3 \%)$ y recibir pedidos por internet (IC $95 \%$ : 40,06\%; 54,14\%).

La mayoría de la pyme que tienen página web solo tienen disponible allí la presentación de la empresa (IC 95\%: 92,08\%; 99,84\%) y el catálogo de productos (IC 95\%: 68,45\%; $85,09 \%$ ) y un poco menos de la mitad recibe pedidos por internet (IC 95\%: 33,67\%; $53,20 \%)$.

Las pyme que utilizan medios sociales utilizan principalmente redes sociales (IC 95 \%: 97,64\%; $100 \%$ ) aunque aproximadamente una de cada cinco empresas utilizan sitios web que comparten contenido multimedia (IC 95\%: 12,30\%; $26,10 \%$ ) y blogs/microblogs (IC $95 \%$ : 11,61 \%; $25,19 \%)$.

La mayoría de las pyme que utilizan los medios sociales lo hacen para comunicarse con sus clientes (IC 95\%: 89,31\%; 97,89\%) y desarrollo de la imagen corporativa (IC $95 \%$ : $85,24 \%$; 95,56\%).

La mayoría de las pyme que utilizan medios sociales consideran que estos son importantes 
$(31,75 \%)$ o muy importantes $(49,21 \%)$ para el desarrollo del negocio.

En cuanto a la inversión en TIC (ordenadores y equipos periféricos, componentes electrónicos y otros bienes y componentes TIC), el 61,93 \% de las pyme invirtió en el 2013 menos de cinco millones de pesos y el 30,41\% invirtió entre cinco y menos de veinte millones de pesos en TIC.

\section{Comentarios}

En general el uso de software especializado diferente a software contable sigue siendo muy limitado en las pyme del sector industrial del $\mathrm{AMB}$, pese a los beneficios que pueden representar los paquetes de planificación de recursos empresariales, diseño, simulación, pronósticos, etc.

Por otra parte, el uso de las distintas herramientas ofimáticas es muy variable dependiendo del subsector.

Las pyme están desaprovechando la oportunidad de utilizar la página web, los medios sociales y las plataformas electrónicas para interactuar más con los clientes y desarrollar actividades de promoción y venta.

El nivel de inversión de las pyme en TIC es muy limitado y no acorde con la necesidad que tienen de ser más productivas y competitivas.

Este trabajo establece la línea base para diseñar e implementar proyectos de acompañamiento para que las pyme del sector industrial del AMB aumenten su productividad y competitividad mediante una mayor integración de las TIC al desarrollo de sus actividades primarias y de apoyo.

\section{Referencias}

CaZorzi, A. (2011). Las TIC en el desarrollo de la pymes. Algunas experiencias de América Latina.

Cámara de Comercio de Bucaramanga. (2015). Industria en Santander No. 118. Retrieved April 16, 2015 , from http://www.camaradirecta.com/temas/document os pdf/informes de actualidad/2015/industria.pdf

Cardona, M., Kretschmer, T., \& Strobel, T. (2013). ICT and productivity: Conclusions from the empirical literature. Information Economics a nd Policy, 25(3), 109125. http://doi.org/10.1016/j.infoecopol.2012.12.002

Colombo, M. G., Croce, A., \& Grilli, L. (2013). ICT services and small businesses productivity gains: An analysis of the adoption of broadband Internet technology. Information Economics and Policy, 25(3), 171189. http://doi.org/10.1016/j.infoecopol.2012.11.001

ComptiA. (2007). Los beneficios económicos y sociales del uso de las TIC - Una Valoración y Guía de Políticas Para América Latina y el Caribe.

Dos Reis, R. A., \& Do Carmo Duarte Freitas, M. (2014). Critical factors on information technology acceptance and use: An analysis on small and medium Brazilian clothing industries. Procedia Computer Science, 31(Itqm), 105114. http://doi.org/10.1016/j.procs.2014.05.250 
Gálvez Albarracín, E. J. (2014). Tecnologías de información y comunicación, e innovación en las MIPYMES de Colombia. Cuadernos de Administracion.

Gálvez Albarracín, E. J., Riascos Erazo, S., \& Contreras Palacios, F. (2014). Influencia de las tecnologías de la información y comunicación en el rendimiento de las micro, pequeñas y medianas empresas colombianas. Estudios Gerenciales, $30(133), 355364$.

Martin, F. M., Ciovica, L., \& Cristescu, M. P. (2013). Implication of Human Capital in the
Development of SMEs through the ICT Adoption. Procedia Economics and Finance, 6(13), 748753. http://doi.org/10.1016/S22125671(13)00198-6

Porter, M. E. (2011). ¿Qué es la estrategia? Harvard Business Review, 89(11), 100117.

Rodríguez Melo, G. E. (2011). Apropiación y masificación de las tecnologías de la información $\mathrm{y}$ las comunicaciones (TIC) en las cadenas productivas como determinante para la competitividad de las Mipyme. Criterio Libre, 9(15), 213230. 